\title{
Demonstration of pulse controlled all-optical switch/modulator
}

\author{
Osman Akin* and M. S. Dinleyici \\ Izmir Institute of Technology, Electrical \& Electronics Engineering, Gulbahce Koyu, Urla, 35430 Izmir, Turkey \\ *Corresponding author: osmanakin@iyte.edu.tr
}

Received July 10, 2013; revised November 24, 2013; accepted January 27, 2014;

posted January 30, 2014 (Doc. ID 193368); published March 10, 2014

\begin{abstract}
An all-optical pulse controlled switch/modulator based on evanescent coupling between a polymer slab waveguide and a single mode fiber is demonstrated. Very fast all-optical modulation/switching is achieved via Kerr effect of the nonlinear polymer placed in the evanescent region of the optical fiber. Local refractive index perturbation $\left(\Delta n=-1.45612 \times 10^{-5}\right)$ on the thin film leads to $0.374 \mathrm{nW}$ power modulation at the fiber output, which results in a switching efficiency of $\approx 1.5 \%$. (C) 2014 Optical Society of America

OCIS codes: (060.2340) Fiber optics components; (130.4815) Optical switching devices; (190.4360) Nonlinear optics, devices; (190.3270) Kerr effect.

http://dx.doi.org/10.1364/OL.39.001469
\end{abstract}

All-optical light manipulation is one of the most prominent goals in photonic communication systems targeting data transmission rates at Tbits/s. Applications include all optical devices based on optical patterns created in nonlinear media [1], modulators [2], optically controlled coupler based switches [3], grating based in-fiber optical switches [4], and optical gates based on third-order nonlinearity [5] for integrated photonic circuits.

Nonlinear optical polymers possess a great potential for use in photonic systems. Along with fundamental research on novel materials, intensive applied research is carried out for implementation of these materials into switching devices for all optical applications [6].

The refractive index of the dye doped polymeric film (waveguide) can locally be altered by using the thirdorder nonlinear optical phenomenon, known as the Kerr effect. Kerr-type processes in polymeric materials offer great advantages in terms of high nonlinearity and processing simplicity [?].

In this Letter, we demonstrate an all-optical switch/ modulator based on a polymeric film-single mode fiber (SMF) coupling structure that evanescently couples the light from a side polished single mode fiber to the nonlinear polymer slab waveguide, as illustrated in Fig. 1(a). Applying an optical control pulse on the polymeric film results in local refractive index perturbation due to Kerr effect. This effect gives rise to power transfer from the propagating modes into the radiation and slab modes that can be explained as pulse-controlled power transfer from the fiber. Refractive-index modulation arising from intensity-dependent Kerr effect becomes the shape of the control beam intensity profile since the beam is freely coupled from the air. The high speed of the switching device is due to the instantaneous response time of the material to the applied control signal. The photon energy of the excitation source at $532 \mathrm{~nm}$ is $2.33 \mathrm{eV}$, and it is far away from the band gap of the polymeric material used in the experiment, which is about $5.3 \mathrm{eV}$ [8]. Therefore, the nonlinear effect is transient and limited with the pulse duration [9].

The coupling between a fiber and a high index planar waveguide has been theoretically investigated by Dinleyici and Patterson [10] using the vector modal solution method. Alternatively, this structure has also been described by the generalized analysis method using the coupled mode theory [11], including a four-layer model of side polished fiber structures. A fiber/slab coupler has also been proposed and numerically investigated using the coupled mode theory [12] and beam propagation methods [13].

A basic structure of the experimental device is shown in Fig. 1(b). This is a structure formed by polishing the single mode fiber (Corning HI1060) cladding, replaced with thin polymeric film. The length of the fiber is $2 \mathrm{~m}$ and the polished cladding part is created just in the middle of the fiber. The flattened region is about $10 \mathrm{~mm}$ and the distance between core and flat side of the fiber is approximately $9.22 \mu \mathrm{m}$, which is shown in Fig. 1(c).

The nonlinear slab layer (polymeric film) is prepared according to the recipe that has been previously introduced by He et al. [14]. The host polymer PVA (Sigma Aldrich, Mol. Wt. 30 000-70 000) and dye MR (Sigma Aldrich) were dissolved in distilled water separately and then filtered to eliminate the particles. The solution was mixed and stirred for at least 12 hours. Before being used in the fiber device, the mixture was coated onto microscope glass in order to analyze the absorption, linear, and third-order nonlinear properties of the thin film

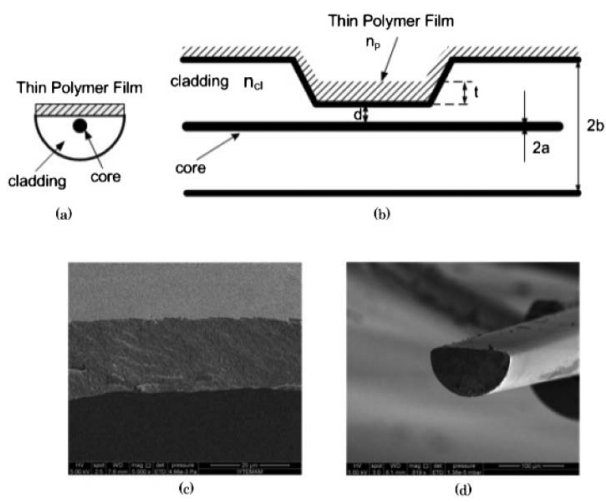

Fig. 1. (a) Cross-section of the polished fiber. (b) Coupler structure geometry. (c) SEM image of the polished part of the fiber. (d) SEM image of the thin film. 
at $532 \mathrm{~nm}$. The linear refractive index of thin film has been measured experimentally using the Fresnel diffraction-based nondestructive measurement method [15] and estimated as $n=1.4995$. The tolerance of the measurement method is given as $5 \times 10^{-5}$. The Kerr coefficient was measured $n_{2}=-4.616 \times 10^{-11} \mathrm{~cm}^{2} / \mathrm{W}$ using the Z-scan experimental technique and found as $n_{2}=$ $-4.02 \times 10^{-11} \mathrm{~cm}^{2} / \mathrm{W}$. In this study, a diode-pumped solid-state (DPSS) laser with wavelength of $\lambda=$ $532 \mathrm{~nm}$ was used, and a pulse duration of about $15 \mathrm{~ns}$ was measured for the beam. Peak intensity at the focus of the objective lens was found as $I_{0}=$ $1.846 \times 10^{8} \mathrm{~W} / \mathrm{cm}^{2}$, and the spot diameter at the focus was measured as $1 \mu \mathrm{m}$.

At the beginning, the presence of external polymeric film on the guided mode is investigated. The sidepolished SM fiber without a polymeric layer is excited with a $632.8 \mathrm{~nm}, 1.5 \mathrm{~mW} \mathrm{He}-\mathrm{Ne}$ laser. The light that emerged from this laser is coupled into the fiber with objective lens having NA value of 0.11 and far-field radiation at the fiber end is recorded by CCD with pixel size of $11 \mu \mathrm{m} \times 11 \mu \mathrm{m}$, which is shown in Fig. 2 . In this case, the power at the output of the fiber is measured as $1.23 \mu \mathrm{W}$ using a power meter. Subsequently, the PVAMR dye mixture is coated onto the polished part of the fiber by the Doctor Blade method by moving a glass frame along the surface. Afterward, the polymeric layer is dried approximately $24 \mathrm{~h}$ at room temperature. After coupling the light emerged from the aforementioned laser into the fiber device again, nearly all of the light is unbounded in the transverse plane and radiated into the thin film; thus a few $\mathrm{nW}$ of power is measured at the fiber output. Because of a high index contrast between refractive indices of polymeric thin film and SMF's cladding, thin film behaves like a sink [16].

Because the radiated power is extremely sensitive to the external film refractive index, the index contrast between these two media should be decreased to reduce the power radiated from SMF. To increase the refractive index of the nonlinear material and enhance the thirdorder nonlinearity of the film [1] $,[\underline{18}], 20 \mathrm{~nm} \mathrm{SiO}_{2}$ particles with refractive indices of 1.46 at $532 \mathrm{~nm}$ (Alfa Aesar-Pr. No. 12727) were added to the mixture of PVA and dye solutions such that nanoparticles and PVA weight equally $\left(\mathrm{SiO}_{2}-\% 7.35\right.$, PVA-\%7.42, methyl red-\% 0.014). The resulting film, as shown in Fig. 1(d), has an approximate thickness of $17 \mu \mathrm{m}$. In this case, the magnitude of the Kerr coefficient has risen to $n_{2}=-4.616 \times 10^{-11} \mathrm{~cm}^{2} / \mathrm{W}$, which is obtained by z-scan experiment, and linear refractive index was estimated to
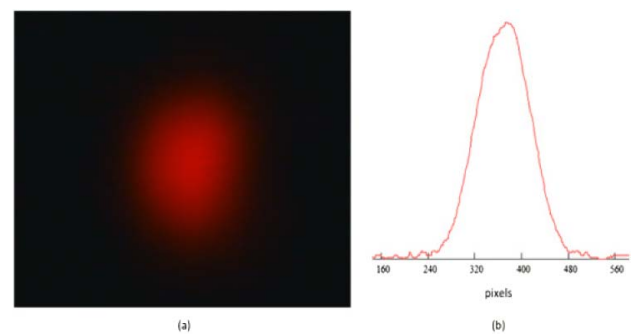

Fig. 2. (a) Far field profile of the beam radiated from fiber end. (b) $2 \mathrm{D}$ profile of the measured data. be about 1.48. In order for a nonlinear material to be useful in optical signal processing devices, two types of figure of merits given by letters $\mathrm{W}$ and $\mathrm{T}$ are defined by Stegeman [19]. The figure of merit $\mathrm{W}$ is related with the linear absorption, and the effect of the nonlinearity must be stronger than linear absorption, a case expressed by merit factor $W=\Delta n / \alpha_{0} \lambda>1, \Delta n=n_{2} I$ is the induced index change and $\alpha_{0}$ is the linear absorption. The $\mathrm{T}$ factor represents the effect of the two photon absorption, and this effect must be weaker than nonlinearity, which implies $T=2 \beta_{2} \lambda / n_{2}<1$, where $\beta$ is the two photon absorption coefficient [20]. The results obtained in the experimental investigation fulfill the figure of merits for polymeric materials to be used in photonic systems [21]. Figure of merit $\mathrm{W}$ is estimated as $W=2.05>1$ and figure of merit $T \cong 0$ [14].

In order to form the fiber-switching device, a PVA-MR dye mixture with nanoparticles is coated onto the polished fiber, as illustrated in Fig. 1(b). When the light of the CW $632.8 \mathrm{~nm} \mathrm{l}$ is launched into the fiber and only a single mode is excited, then the power at the fiber output has increased up to $25 \mathrm{nW}$, which refers to $47 \mathrm{~dB}$ insertion loss. The measured power is enough to test the switching capability of the proposed device.

The basic structure of the experimental setup is illustrated in Fig. 3. The control pulse has 14 ns pulse duration (FWHM) at $3 \overline{5} \mathrm{kHz}$ repetition rate, shown in Fig. 4(a), and the spot size of the Gaussian-like beam at the laser output is measured as $1.43 \mathrm{~mm}$ [Fig. 4(b)]. The control pulses are focused to an elliptical spot onto the slab waveguide at normal incidence by using plano convex and objective lenses (NA: $0.11, \times 4$ ). The ellipsoidal spot has a minor axis of about $0.5 \mathrm{~mm}$, the major axis was about $2 \mathrm{~mm}$, and the arrangement was made such that the major axis coincides with the optical axis of the fiber core. It should be noted that the spot size of the beam plays an important role in switching the mechanism since these temporal and spatial profiles cast into a refractive index modulation. It is desirable to be as large as possible, but also it

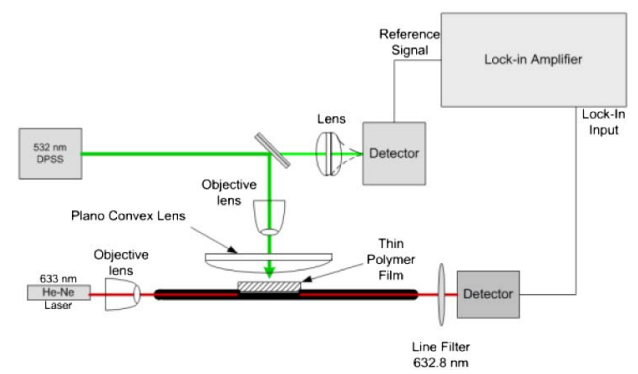

Fig. 3. Schematic of the experimental setup.

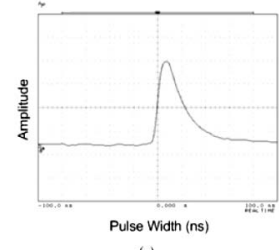

(a)

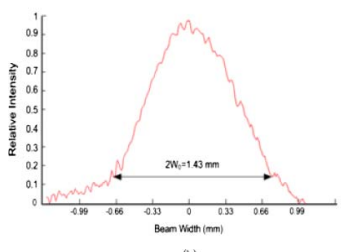

(b)
Fig. 4. Control beam (a) temporal pulse profile (b) spatial beam profile. 
should contain enough power to harvest the Kerr nonlinearity. However, although the applied beam spot size is in elliptical form with an axis of about $0.5 \mathrm{~mm} \times 2 \mathrm{~mm}$, the influence of the beam is limited with the fiber core size with a diameter of $5.3 \mu \mathrm{m}$. This is the effective switching (ES) area on the device, and its size can be defined as $5.3 \mu \mathrm{m} \times 2 \mathrm{~mm}$.

The power variations due to control pulses are measured using a high speed Silicon PIN detector (Newport 818BB21) placed at the end of the fiber and connected to lock-in amplifier (Stanford Research-SR830), as shown in Fig. 3. The noise floor of the lock-in amplifier is in the order of a few $\mathrm{pW}$ range, based on experimental (environmental) condition. In order to synchronize the system, the reference input of the lock-in amplifier was obtained from the output of another detector having the same response time. Additionally, a $632.8 \mathrm{~nm}$ laser line filter (FWHM $=10 \mathrm{~nm}, \geq 4)$ was used to reject the radiation and power leakage due to $532 \mathrm{~nm}$ control pulse laser.

In order to ascertain the switching/modulating capability of the coupler, fiber/device output is observed with a lock-in amplifier to measure the power variations when the control pulses are applied onto the film slab layer. The switching/modulation is due to controlling the radiation of light from the SM fiber to the polymeric slab layer. When the control pulse is applied on the nonlinear film, the refractive index decreases (due to negative Kerr coefficient), and the amount of light coupled into the slab decreases during the pulse length. This results in power increase at the fiber output.

At the beginning of the switching process, in the absence of control pulse, the fiber output power remains at a constant level of $25 \mathrm{nW}$. Since the lock-in amplifier is operated in the AC mode for all time and there was not any time-varying signal to be locked, it reads zero. Then, the pulse power is consistently increased by tuning DPSS current and/or repetition rate parameters until some power variations were measured at the fiber output. The measurements show that switching begins after some threshold value for the control signal pulse power is reached. When the pulse energy reaches $9.6 \mu \mathrm{J}$, which refers to an average power $P_{\mathrm{av}}=0.1435 \mathrm{~W}$ and peak pulse power $P_{p}=561 \mathrm{~W}$, a refractive index modulation of $\Delta n=-3.4827 \times 10^{-6}$ is obtained in the nonlinear medium and controllable coupling process becomes observable. The power density in the ES area is estimated to be $75.45 \mathrm{~kW} / \mathrm{cm}^{2}$. When the applied control pulse energy reaches $40 \mu \mathrm{J}$, the maximum signal modulation is obtained and the maximum power of $25.374 \mathrm{nW}$ is measured at the fiber output. This power increase corresponds to the $\approx 1.5 \%$ switching efficiency. The average and peak power of the laser pulse train was estimated as $0.6 \mathrm{~W}$ and $2.344 \mathrm{~kW}$, respectively. However, the power density in the ES area was $315.29 \mathrm{~kW} / \mathrm{cm}^{2}$, which results in refractive index modulation $\Delta n=-1.45612 \times 10^{-5}$. The effective beam power that dominates only the ES area and triggers the nonlinearity was estimated to be $0.33 \mathrm{~W}$. The left side of Fig. 5 shows the normalized measured power at the fiber output as a response to the power density of the applied control pulse. When control pulses are applied on the film, the refractive index of the film is locally decreased with the geometry of the beam

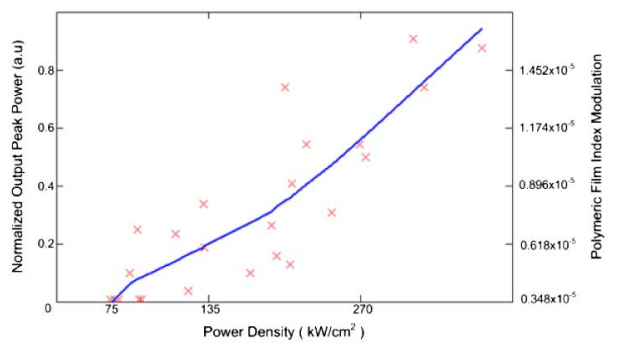

Fig. 5. Peak power variation at fiber output according to the power density.

profile (the film has negative Kerr coefficient), and some of the power propagating in the fiber is transferred to the slab waveguide modes. This is consistent with the theoretical results obtained by Dinleyici and Patterson [10]. At this rate of power density, no thermal deformation is observed, but further increasing the power density in ES area up to $403.52 \mathrm{~kW} / \mathrm{cm}^{2}$ results in disruption in the polymer film. In this case, the lock-in amplifier is not able to lock to the reference signal.

Moreover, the application position of the control pulse is also important for the coupling process, as shown in Fig. 6 . The polished region was divided into three parts and each part, as shown in Fig. 6(a), was illuminated with an elliptical beam with axes of 0.5 and $2 \mathrm{~mm}$. The highest switching efficiency was observed when region 2 is illuminated and the output peak power was about $25.374 \mathrm{nW}$. Although the refractive index modulation remains the same in all three regions, the effect of index modification is small in region 1 and region 3. The main reason for this result is the fiber core-flat side separation "d," illustrated in Fig. 6(c). The parameter "d" determines the amount of power of the evanescent field that will interact with the index-modulated region. When the separation distance "d" is increased, the coupling coefficient is decreasing and the effect of index modulation on the ridge modes is diminishing, and experimental works in this study support the findings of Dinleyici and Patterson [10], Lee [12], and Cooper [21]. Since the index modulation has the ellipsoidal-like shape, rather than covering the whole film, the coupling among the modes (ridge modes, leaky modes and radiation modes) are quite complicated to track with mathematical methods; presumably, the experimental results are accumulation of all these coupling processes. Demonstrated all-optical

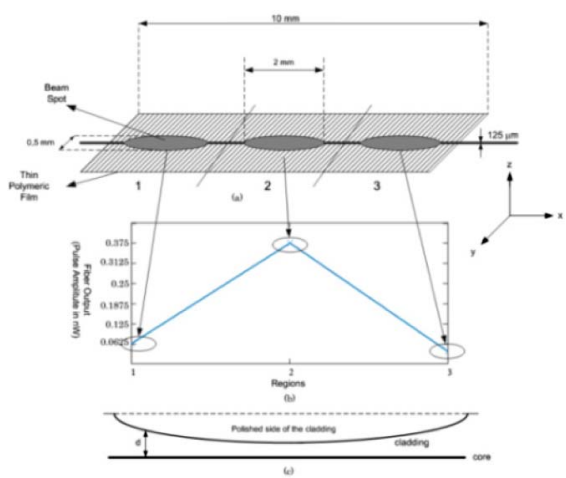

Fig. 6. Switching efficiency dependence on the laser spot position. 
method can be exploited for ultrafast light manipulation applications such as light modulators and/or all-optical switching. Furthermore, operating this fiber near cutoff wavelengths provides lower light power dynamic switching. However, the efficiency is very sensitive to linear refractive index of the polymer; thus, the structure can be optimized in terms of polymeric material refractive index and buffer thickness to increase the modulation contrast at the device output. Furthermore, total power may be increased by using structured coupling between waveguides, which is planned as our next experimental study.

This work was supported by the Scientific and Technical Research Council of Turkey, TUBITAK under project 109E240.

\section{References}

1. A. M. C. Dawes, D. J. Gauthier, S. Schumacher, N. H. Kwong, R. Binder, and A. L. Smirl, Laser Photon. Rev. 4 221 (2010).

2. A. Elezzabi, Z. Han, S. Sederberg, and V. Van, Opt. Express 17, 11045 (2009).

3. P. Sahu, Appl. Opt. 51, 2601 (2012).

4. O. Akin and M. S. Dinleyici, J. Lightwave Technol. 28, 3470 (2010).

5. T. Vo, J. Schröder, R. Pant, M. Pelusi, S. Madden, D. Choi, D. Bulla, B. Luther-Davies, and B. Eggleton, Optical Fiber Communication Conference, OSA Technical Digest (CD) (Optical Society of America, 2011), paper OWG2.
6. B. Block, S. Liff, M. Kobrinsky, M. Reshotko, R. Tseng, I. Ban, and P. Chang, Proc. SPIE 8629, 86290Z (2013).

7. S. A. Haque and J. Nelson, Science 327, 1466 (2010).

8. A. N. Ananth, S. Umapathy, J. Sophia, T. Mathavan, and D. Mangalaraj, Appl. Nanosci. 1, 87 (2011).

9. T. Schneider, D. Wolfframm, and J. Reif, Nucl. Instrum. Methods Phys. Res. Sect. B 166, 809 (2000).

10. M. S. Dinleyici and D. B. Patterson, J. Lightwave Technol. 15, 2316 (1997).

11. L. N. Binh and S. Zheng, J. Opt. Soc. Am. B 18, 1639 (2001).

12. C. H. Lee, C. Kim, J. H. Park, and S. W. Kang, J. Korean Phys. Soc. 54, 69 (2008).

13. C. Kim, C. H. Lee, J. H. Park, and D. M. Choi, J. Korean Phys. Soc. 56, 329 (2009).

14. T. He, Y. Cheng, Y. Du, and Y. Mo, Opt. Commun. 275, 240 (2007).

15. M. S. Dinleyici and C. Sümer, Opt. Commun. 284, 5067 (2011).

16. S. M. Tseng and C. L. Chen, Appl. Opt. 31, 3438 (1992).

17. M. H. Nayfeh, G. Belomoin, N. Barry, and O. Akcakir, "Silicon nanoparticles microcrystal nonlinear optical devices," U.S. patent 6,456,423 (September 24 2002).

18. P. Watekar, S. Moon, A. Lin, S. Ju, and W. T. Han, J. Lightwave Technol. 27, 568 (2009).

19. G. I. Stegeman, Proc. SPIE 1852, 75 (1993).

20. L. Brzozowski and E. H. Sargent, J. Mater. Sci.: Mater. Electron. 12, 483 (2001).

21. M. Cooper and S. Mookherjea, Opt. Express 17, 1583 (2009). 